\title{
Passive Muscle Length Changes Affect Twitch Potentiation in Power Athletes
}

\author{
PAULO GAGO ${ }^{1,2,3}$, MÁRIO CARDOSO MARQUES ${ }^{1,2}$, DANIEL ALMEIDA MARINHO ${ }^{1,2}$, \\ and MARIA M. EKBLOM ${ }^{3,4}$
}

\author{
${ }^{1}$ Sports Sciences Department, University of Beira Interior, Covilhã, PORTUGAL; ${ }^{2}$ Research Center for Sport, Health and \\ Human Development, PORTUGAL; ${ }^{3}$ Biomechanics and Motor Control laboratory, Swedish School of Sport and Health Sciences, \\ Stockholm, SWEDEN; and ${ }^{4}$ Department of Neuroscience, Karolinska Institutet, Stockholm, SWEDEN
}

\begin{abstract}
GAGO, P., M. C. MARQUES, D. A. MARINHO, and M. M. EKBLOM. Passive Muscle Length Changes Affect Twitch Potentiation in Power Athletes. Med. Sci. Sports Exerc., Vol. 46, No. 7, pp. 1334-1342, 2014. Introduction: A conditioning maximal voluntary muscle action (MVC) has been shown to induce postactivation potentiation, that is, improved contractile muscle properties, when muscles are contracted isometrically. It is still uncertain how the contractile properties are affected during ongoing muscle length changes. The purpose of this study was to investigate the effects of a 6-s conditioning MVC on twitch properties of the plantarflexors during ongoing muscle length changes. Methods: Peak twitch, rate of torque development, and rate of torque relaxation, rising time, and half relaxation time were measured from supramaximal twitches evoked in the plantarflexors of 11 highly trained athletes. Twitches were evoked before a 6-s MVC and subsequently on eight different occasions during a 10-min recovery for five different modes: fast lengthening, slow lengthening, isometric, fast shortening, and slow shortening of the plantarflexors. Results: The magnitude and the duration of effects from the conditioning MVC were significantly different between modes. Peak twitch, rate of torque development, and rate of torque relaxation significantly increased for all modes but more so for twitches evoked during fast and slow shortening as compared with lengthening. Rising time was reduced in the lengthening modes but slightly prolonged in the shortening modes. Half relaxation time was significantly reduced for all modes, except fast lengthening. Conclusions: The findings show that the effects of a conditioning MVC on twitch contractile properties are dependent on direction and velocity of ongoing muscle length changes. This may imply that functional enhancements from a conditioning MVC might be expected to be greatest for concentric muscle actions but are still present in isometric and eccentric parts of a movement. Key Words: POTENTIATION, CONCENTRIC, MUSCLE STRENGTH, SPORTS, TWITCH
\end{abstract}

$\mathrm{P}$ ostactivation potentiation (PAP) can be defined as the acute enhancement of muscle contractile properties induced by a maximal (MVC), or near maximal, voluntary contraction $(5,21,24)$. Such contractions have been shown to increase the peak twitch and rate of torque development (RTD) and to decrease the time to peak (TTP) and half relaxation time (HRT) of an electrically evoked twitch in human muscles for up to $10 \mathrm{~min}(5,24,28,33)$. Electrically evoked twitches are used to assess muscle contractile properties because they allow for isolated studies of contractile properties, whereas the assessment of sports performance enhancement after a conditioning task can be caused by many factors, including psychological placebo-like effects. It has been shown in humans that a brief highintensity muscle activation can increase the phosphorylation

Address for correspondence: Maria Ekblom, The Swedish School of Sport and Health Sciences, Box 5626, 11486 Stockholm, Sweden; E-mail: maria.ekblom@gih.se.

Submitted for publication July 2013.

Accepted for publication December 2013.

0195-9131/14/4607-1334/0

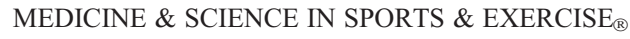

Copyright $\left({ }^{\circ} 2014\right.$ by the American College of Sports Medicine

DOI: 10.1249/MSS.0000000000000245 of the myosin regulatory light chains (37), which can ultimately improve the excitation-contraction coupling and thereby modulate the cross-bridge kinetics $(7,8,40)$. A few authors have shown that the PAP of contractile properties in a twitch is associated with improvements in RTD in voluntary isometric muscle actions (5) and load relationships in concentric muscle actions (4). Studies erroneously ascribe any performance enhancement found after a conditioning muscle action to changes in contractile properties, although they do not assess contractile properties in isolation $(11,22)$. The studies that have assessed PAP via the effects of MVCs on electrically evoked twitches have instead been mainly restricted to twitch properties of muscles contracted isometrically $(5,27,33)$. In most tasks, however, muscles develop force while undergoing length changes. Therefore, it seems important to investigate effects of PAP on contractile properties not only during isometric muscle actions but also in muscles undergoing length changes.

Caterini et al. (10) showed that in mouse muscle, a conditioning muscle stimulation enhanced peak twitch more during muscle shortening than that in a muscle held isometric. No significant changes were found during muscle lengthening. Furthermore, power production was only enhanced during fast shortening. In slow muscle shortening and lengthening, power production was reduced after a conditioning MVC. In contrast 
in humans, Babault et al. (2) concluded that $5 \mathrm{~s}$ after a conditioning MVC, the TTP and the RTD of a quadriceps twitch were significantly enhanced both during passive shortening and lengthening. To assess potentiation of twitches performed during muscle lengthening, these authors performed the MVC with the knee set at $20^{\circ}$, whereas to assess potentiation of twitches performed during shortening, they performed the conditioning $\mathrm{MVC}$ with the knee set at $96^{\circ}$. Although this may be relevant for ensuring that the thixotropy of the muscle was reset to the current muscle length, it may instead be that the conditioning MVCs were not of the same magnitude for shortening and lengthening. In fact, in the same study, it was shown that a twitch response assessed at $56^{\circ}$ showed less potentiation when the conditioning MVC was performed at $20^{\circ}$ compared with when it was performed at $96^{\circ}$. This means that the comparisons between potentiation in passive shortening and lengthening were not entirely valid. Results may have been different if evoked twitch responses assessed during passive shortening and lengthening had been conditioned by an MVC performed at the same muscle length as the evoked twitches. The aim of the current study was to investigate, in athletes, if and how the amplitude and duration of changes in twitch properties seen after an isometric conditioning MVC are dependent on muscle action types (isometric, shortening, or lengthening) and muscle length change velocities.

\section{METHODS}

Subjects. Eleven highly trained male athletes (eight national level sprinters and three jumpers (age $=21.4 \pm 2.6 \mathrm{yr}$, height $=186 \pm 6.1 \mathrm{~cm}$, mass $=80.9 \pm 7.9 \mathrm{~kg}$, body mass index $=23.3 \pm 1.5 \mathrm{~kg} \cdot \mathrm{m}^{-2}$ ) recruited from athletics clubs in Stockholm participated in the present study. Subjects were free from previous ankle injury, and all tests were performed on the plantarflexor muscles of their right leg. All subjects provided their informed written consent before participation in the study. The study was approved by the local ethics committee, and all procedures adhered to the declaration of Helsinki.

Experimental procedures. The experiment lasted for approximately $2.5 \mathrm{~h}$, beginning with $10 \mathrm{~min}$ of submaximal ergometer cycling to warm up. While resting, electrodes were placed on the subjects (see electrical stimulation and EMG protocol). They were then positioned in the isokinetic dynamometer (Isomed 2000; D\&R Ferstl Gmbh, Henau, Germany), lying prone with their arms and hands at the side of their body. Their shoulders, hips, legs, and right foot were adequately fixated, paying special attention to securely strapping the foot into the foot attachment of the Isomed 2000. The axis of the ankle joint was aligned with the rotational center of the dynamometer's shaft, and a gravity correction of the torques created by the passive weight of the foot was executed using Isomed 2000 built-in software. After these preparations, electric stimulation intensity was set (see electrical stimulation). After resting for $10 \mathrm{~min}$ to abolish any kind of remaining potentiation that could influence the data, five separate protocols were executed. Each protocol consisted of three supramaximal twitches, termed control twitches (see electrical stimulation), followed by a 6-s maximal isometric contraction (6-s MVC) conditioning phase. Finally, during the proceeding 10 min of recovery, eight subsequent twitches were evoked (Fig. 1A). One protocol was performed with the foot at $90^{\circ}$ (ISO mode), two performed during passive lengthening (at velocities of $30^{\circ} \cdot \mathrm{s}^{-1}$ and $\left.60^{\circ} \cdot \mathrm{s}^{-1}\right)\left(\mathrm{LEN}_{\text {fast }}\right.$ and $\mathrm{LEN}_{\text {slow }}$ modes $)$, and two during passive shortening (at velocities of $30^{\circ} \cdot \mathrm{s}^{-1}$ and $\left.60^{\circ} \cdot \mathrm{s}^{-1}\right)\left(\mathrm{SHO}_{\text {fast }}\right.$ and $\mathrm{SHO}_{\text {slow }}$ modes). For each subject, the order of the five protocols was randomized. After $10 \mathrm{~min}$ of recovery, approximately $2 \mathrm{~min}$ of further rest was given before the next protocol was initiated.

Electrical stimulation. Electrical stimulation was applied to the posterior tibial nerve using a single rectangular

A

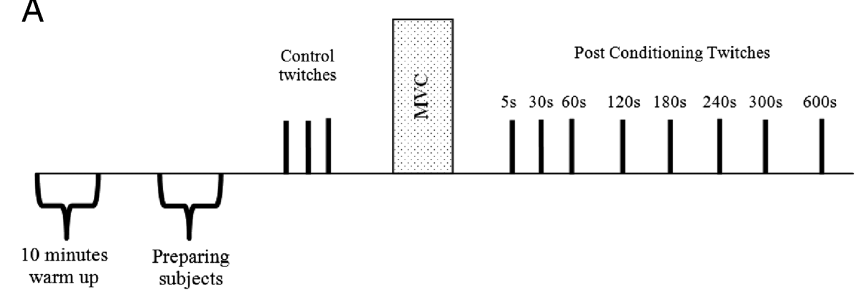

$\mathrm{B}$

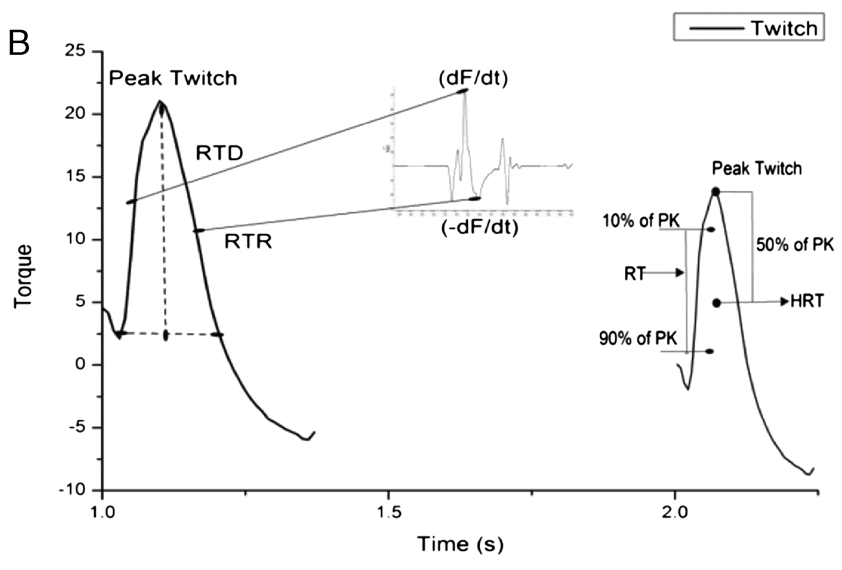

C

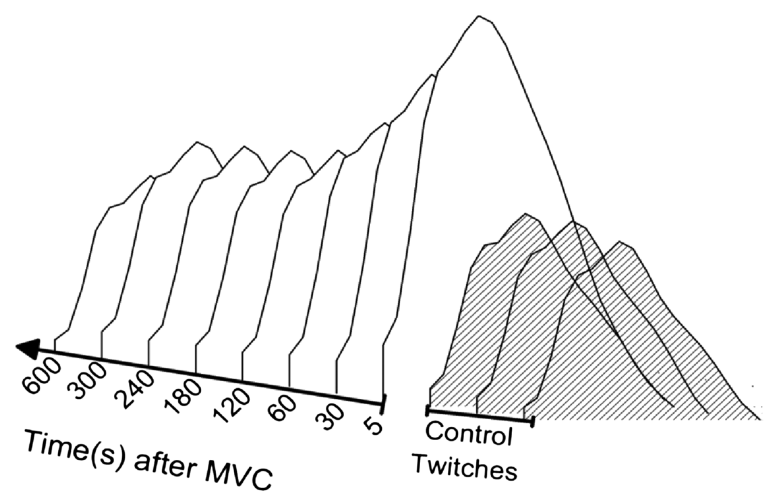

FIGURE 1-A, Representation of the overall study protocol. B, Example of an individual twitch showing the analyzed twitch parameters during ongoing muscle length changes: peak twitch (PT), rate of torque development (RTD), rate of torque relaxation (RTR), rising time from $10 \%$ to $90 \%$ of the peak twitch $\left(\mathrm{RT}_{10-90}\right)$, and half relaxation time (HRT). C, Raw data with an example of the effects of a 6-s MVC on plantarflexor twitches in the isometric mode. 
pulse $(1 \mathrm{~ms})$ delivered by a Digitimer stimulator (model DS7A; Digitimer, Hertfordshire, UK). A small cathode electrode (blue sensor, 7-mm diameter, $\mathrm{Ag}-\mathrm{AgCL}$ Medicotest, Denmark) was positioned in the popliteal fossa after a manual location of the best stimulation zone in that area, using a custom-made stimulating pen. The anode (a $100 \times 50-\mathrm{mm}$ carbon rubber electrode; Cefar Medical, Sweden) was placed then taped on the anterior surface of the knee, proximal to the patella. Each subject was initially familiarized with several submaximal electrical stimuli of progressively increased intensity until the compound muscle action potential (M-wave), and the mechanical twitch reached their maximal values. The stimulation intensity was then further increased by $20 \%$ to a supramaximal intensity used in the subsequent protocol. Stimulations were always delivered with the foot at $90^{\circ}$ for the isometric protocol, and at angles previously determined during pilot trials, making sure that the peak twitch occurred as the foot moved through $90^{\circ}$ for the SHO and LEN modes. These settings resulted in a peak twitch angle variation between modes of $0.68^{\circ} \pm 2.24^{\circ}$, which is in line with the values presented by Gravel et al. (17).

EMG measurements. EMG signals were recorded using circular electrodes (Blue Sensor 7-mm diameter, AgAgCL Medicotest) positioned in a belly tendon configuration $(2 \mathrm{~cm}$ below the bifurcation of the gastrocnemius muscles and in line with the Achilles tendon) on the soleus muscle and along the belly of the tibialis anterior muscle. A single ground electrode was positioned over the head of the fibula. Low impedance at the skin-electrode interface was obtained by shaving and cleaning the skin with alcohol. The EMG signals were sampled at a rate of $5 \mathrm{kHz}$, amplified 200 times (NL 824; Digitimer), band pass filtered (30 Hz-1 kHz) (NL 125; Digitimer), and converted to digital data using a 16-bit Power 1401 and Spike2 data collection system (version 6.0; Cambridge Electronic Design, UK). Using the soleus EMG signal, the peak-to-peak amplitude of the $\mathrm{M}$ wave associated with each twitch response was measured to ensure the effective stimulus of the nerve was not altered during the protocol.

Mechanical measurements. Torque about the ankle was measured by the isokinetic dynamometer Isomed 2000 (D\&R Ferstl Gmbh), which also controlled the position and angular velocity of the foot. The torque signal was analogto-digital converted and sampled together with the position and EMG data by a power 1401 (CED, Cambridge, England) in Spike2 software (Spike2, version 6.0; CED). The angular impulse of the conditioning MVC was calculated as the area under the torque-time curve from $80 \%$ of the peak for the rising and falling of the curve. The torque signal produced as a result of the evoked twitch was analyzed using the same software to extract the following variables: peak twitch, measured as the difference between the maximal twitch torque value and the torque value at the time of the proximal peak of the Soleus M-wave; HRT, measured as the time from twitch peak torque to the return to $50 \%$ of peak torque; maximum RTD and rate of torque relaxation (RTR), measured as the peak of the first derivate of the development of torque $(d F / d t)$ and as the peak of the first derivate of decline of torque $(-d F / d t)$, respectively; and rising time, measured as the time between $10 \%$ and $90 \%$ of the peak twitch torque on the ascending side of the twitch (Figs. 1B and 1C).

Statistical analyses. Statistical analysis was performed using Statistica (Version 10; StatSoft Scandinavia AB, Uppsala, Sweden). Normality tests were performed using Shapiro-Wilks $W$-tests. A two-way repeated-measures ANOVA was used with the factors time (before and at different delays after the conditioning MVC) and mode (ISO, $\mathrm{LEN}_{\text {fast }}, \mathrm{LEN}_{\text {slow }}, \mathrm{SHO}_{\text {fast }}$, and $\mathrm{SHO}_{\text {slow }}$ ). Wherever a significant main effect or an interaction of factors was found, a Tukey HSD post hoc test was applied. Differences were considered as significant at $P<0.05$. Furthermore, intraclass correlation coefficients $\left(\mathrm{ICC}_{\mathrm{c}}\right)$ were calculated to assess consistency within the control twitches for each parameter and mode and to confirm consistency of the conditioning MVC between modes.

\section{RESULTS}

Conditioning MVC. The mean of the area under the torque-time curve of the conditioning MVC was $1160.48 \pm$ $50.9 \mathrm{~N} \cdot \mathrm{m} \cdot \mathrm{s}$, with no significant differences between modes $(F=1.280, P=0.294)$. The $\mathrm{ICC}_{\mathrm{c}}$ of the conditioning MVC was 0.767 , indicating that the conditioning MVCs were consistent between modes.

Peak twitch. $\mathrm{ICC}_{\mathrm{c}}$ for the control twitch peak twitch ranged between 0.930 and 0.996 in the different modes. The absolute value of the peak twitch was significantly different between modes $\left(F_{4,40}=190.7\right)$, with peak twitch being greater for LEN than SHO. Furthermore, there was a significant interaction between time and mode $\left(F_{32,320}=13.3\right)$, indicating that the change in peak twitch over time differed between modes. Post hoc tests showed that compared with the control twitches, the peak twitch was significantly enhanced up to 4 min after the conditioning MVC for all the angular velocities in the SHO and LEN modes, whereas in the ISO mode, the peak twitch was significantly enhanced up to 5 min after the MVC.

Mode-specific peak twitch PAP (expressed as increase in percent of control twitch values) at different delays after the conditioning MVC is displayed in Figure 2. Peak twitch PAP differed significantly between modes $\left(F_{4,40}=68.2\right)$, and relative changes over time also varied significantly between modes $\left(F_{28,280}=61.6\right)$. The average (mean of all time points) peak twitch PAP after the MVC was highest in the $\mathrm{SHO}_{\text {fast }}$ mode $(37.1 \% \pm 1.2 \%)$ and lowest in the $\mathrm{LEN}_{\text {fast }}$ mode $(9.7 \% \pm 9.4 \%)$. Post hoc tests showed significant differences in peak twitch PAP between $\mathrm{LEN}_{\text {fast }}$ and $\mathrm{LEN}_{\text {slow }}$ at $5 \mathrm{~s}$ after MVC only (Fig. 2). For the same angular velocity, peak twitch PAP differed significantly between SHO and LEN from $5 \mathrm{~s}$ to 2 min after the conditioning MVC (Fig. 2). Finally, in the ISO mode, the peak twitch PAP was $67.0 \% \pm 21.6 \%$ at $5 \mathrm{~s}$ and $11.0 \% \pm 5.8 \%$ at $5 \mathrm{~min}$ after the MVC, sustaining significantly higher values than that in the 


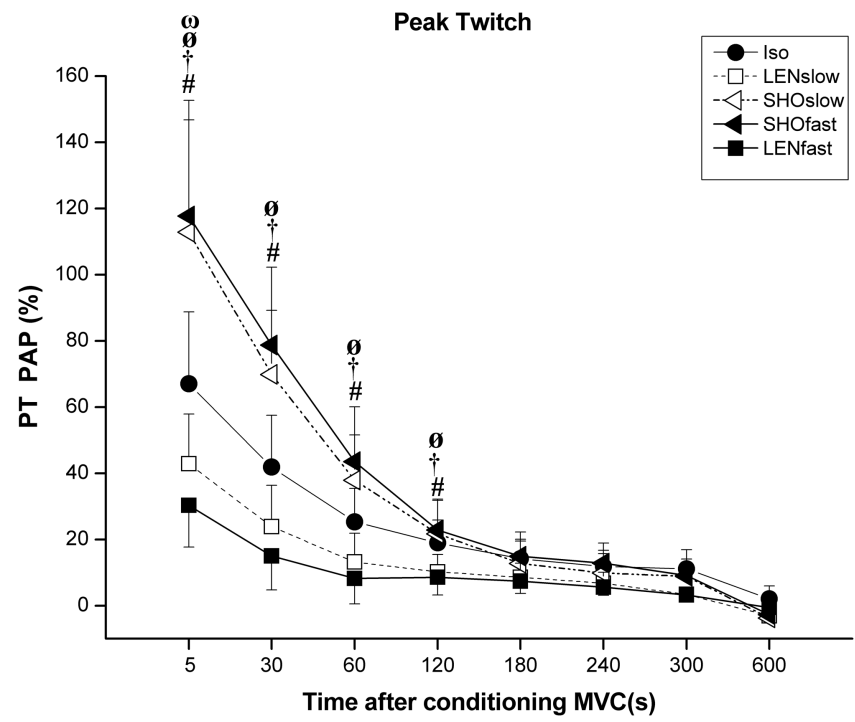

FIGURE 2-Peak twitch (PT) PAP (\% increase from control twitch values) at different delays after the conditioning $M V C$ for the five different modes. Significant $(P<0.05)$ differences in degree of peak twitch PAP are as follows: \#differences between fast lengthening ( $\left.\mathbf{L E N}_{\text {fast }}\right)$ and fast shortening $\left(\mathrm{SHO}_{\text {fast }}\right)$, †differences between slow lengthening $\left(\mathrm{LEN}_{\text {slow }}\right)$ and slow shortening $\left(\mathrm{SHO}_{\text {slow }}\right)$, Ødifferences between isometric and any other mode, and wdifferences between velocities within the lengthening modes.

$\mathrm{LEN}_{\text {fast }}$ and $\mathrm{LEN}_{\text {slow }}$ modes but significantly lower values than that in the $\mathrm{SHO}_{\text {fast }}$ and $\mathrm{SHO}_{\text {slow }}$ modes for up to $2 \mathrm{~min}$ after the MVC (Fig. 2).

RTD. $\mathrm{ICC}_{\mathrm{c}}$ for the control twitch RTD ranged between 0.948 and 0.994 in the different modes. The absolute values of the RTD were significantly different between modes $\left(F_{4,40}=205.0\right)$, with higher RTD in LEN than SHO. An interaction between mode and time $\left(F_{32,320}=95.6\right)$ was also identified and post hoc tests showed that compared with the control twitches, the RTD was significantly enhanced for $3 \mathrm{~min}$ for SHO, 2 min for ISO and $\mathrm{LEN}_{\text {slow }}$, and 4 min for $\mathrm{LEN}_{\text {fast }}$.

RTD PAP (expressed as increase in percent of control twitch values) at different delays after the conditioning MVC are displayed in Figure 3. RTD PAP differed significantly between modes $\left(F_{4,40}=12.5\right)$, and an interaction between mode and time was also identified $\left(F_{28,280}=11.0\right)$. The average RTD PAP was highest in the $\mathrm{SHO}_{\text {slow }}$ mode $35.7 \% \pm$ $37.47 \%$ and lowest in the $\mathrm{LEN}_{\text {slow }}$ mode $20.2 \% \pm 26.76 \%$ (Fig. 3). Post hoc tests showed no significant differences in RTD PAP between $\mathrm{LEN}_{\text {fast }}$ and $\mathrm{LEN}_{\text {slow }}$, or $\mathrm{SHO}_{\text {fast }}$ and $\mathrm{SHO}_{\text {slow }}$ (Fig. 3). For the slow angular velocity modes, RTD PAP values between SHO and LEN differed significantly from $5 \mathrm{~s}$ to $3 \mathrm{~min}$ after the conditioning MVC, whereas at the fast angular velocity, they differed from $5 \mathrm{~s}$ to $1 \mathrm{~min}$ (Fig. 3). Regarding the ISO mode, the RTD PAP was $102.2 \% \pm 33.3 \%$ at $5 \mathrm{~s}$ and $19.9 \% \pm 18.2 \%$ at $2 \mathrm{~min}$ after MVC, sustaining significantly higher values than that in the $\mathrm{LEN}_{\text {fast }}$ and $\mathrm{LEN}_{\text {slow }}$ modes up to $1 \mathrm{~min}$ after the MVC (Fig. 3).

Rate of torque relaxation. $\mathrm{ICC}_{\mathrm{c}}$ for the control twitch RTR ranged between 0.919 and 0.984 in the different modes. The absolute values of the RTR were significantly different between modes $\left(F_{4,40}=50.1\right)$, with RTR being higher for LEN than SHO and with a significant interaction between time and mode $\left(F_{32,320}=5.4\right)$. Post hoc tests showed that compared with the control twitches, the RTR was significantly enhanced for up to $1 \mathrm{~min}$ after MVC for $\mathrm{SHO}_{\text {fast }}, 2 \mathrm{~min}$ for $\mathrm{SHO}_{\text {slow }}$ and $\mathrm{LEN}_{\text {slow }}$, and $4 \mathrm{~min}$ for ISO and $\mathrm{LEN}_{\text {fast }}$.

RTR PAP (expressed as increase in percent of control twitch values) at different delays after the conditioning MVC are displayed in Figure 4. RTR PAP differed significantly between modes $\left(F_{4,40}=6.0\right)$, and an interaction was seen between mode and time $\left(F_{28,280}=12.2\right)$. RTR PAP was highest in the $\mathrm{SHO}_{\text {slow }}$ mode $(30.2 \% \pm 34.24 \%)$ and lowest in the $\mathrm{LEN}_{\text {slow }}$ mode $(15.6 \% \pm 16.94 \%)$. Post hoc tests showed significant differences between $\mathrm{SHO}_{\text {slow }}$ and $\mathrm{SHO}_{\text {fast }}$ only at $5 \mathrm{~s}$ after MVC (Fig. 4). For the same angular velocity, SHO and LEN values differed significantly from $5 \mathrm{~s}$ to $1 \mathrm{~min}$ after the conditioning MVC (Fig. 4). Finally, in the ISO mode, the RTR PAP was $66.9 \% \pm 23.5 \%$ at $5 \mathrm{~s}$ and $11.6 \% \pm 8.9 \%$ at $4 \mathrm{~min}$ after MVC, sustaining significantly higher values than that in the $\mathrm{LEN}_{\text {fast }}$ and $\mathrm{LEN}_{\text {slow }}$ modes for up to $1 \mathrm{~min}$, and significantly lower values than that in the $\mathrm{SHO}_{\text {slow }}$ mode for up to $30 \mathrm{~s}$ after MVC. No significant differences were identified between the ISO mode and the $\mathrm{SHO}_{\text {fast }}$ mode (Fig. 4).

Rising time ${ }_{10-90} \mathrm{ICC}_{\mathrm{c}}$ for the control twitch rising time $_{10-90}$ ranged between 0.875 and 0.990 in the different modes. The absolute values of the rising time ${ }_{10-90}$ were significantly different between modes $\left(F_{4,40}=159.4\right)$, with longer rising time ${ }_{10-90}$ for LEN than SHO. An interaction was found between time and mode $\left(F_{32,320}=5.4\right)$. Post hoc

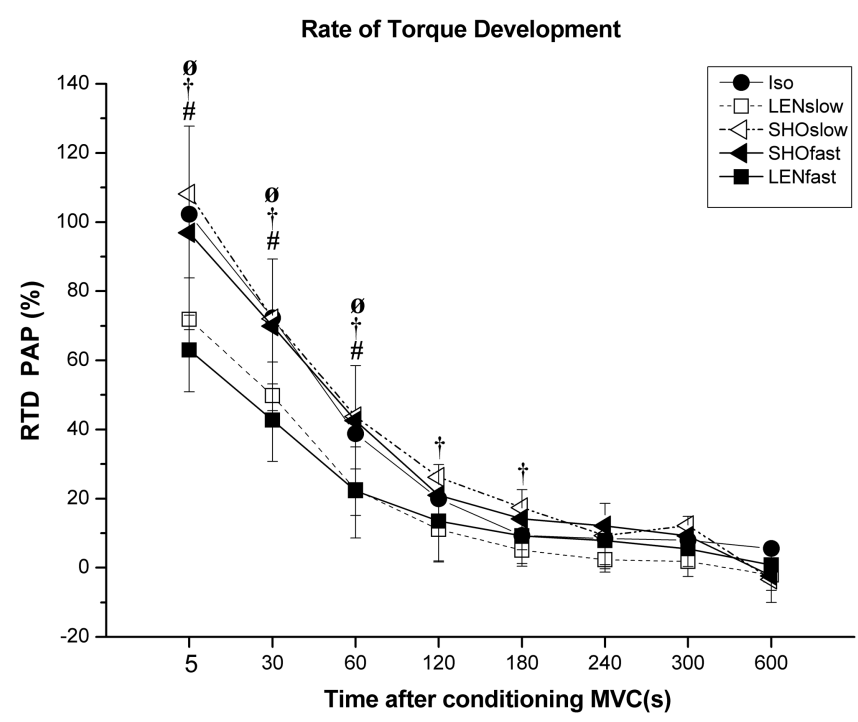

FIGURE 3-Rate of torque development (RTD) PAP (\% increase from control twitch values) at different delays after the conditioning MVC for the five different modes. Significant $(P<0.05)$ differences in degree of peak twitch RTD are indicated by the following: \#differences between fast lengthening $\left(\mathrm{LEN}_{\text {fast }}\right)$ and fast shortening $\left(\mathrm{SHO}_{\text {fast }}\right)$, †differences between slow lengthening $\left(\mathrm{LEN}_{\text {slow }}\right)$ and slow shortening $\left(\mathrm{SHO}_{\text {slow }}\right)$, and Ødifferences between isometric and any other mode. 


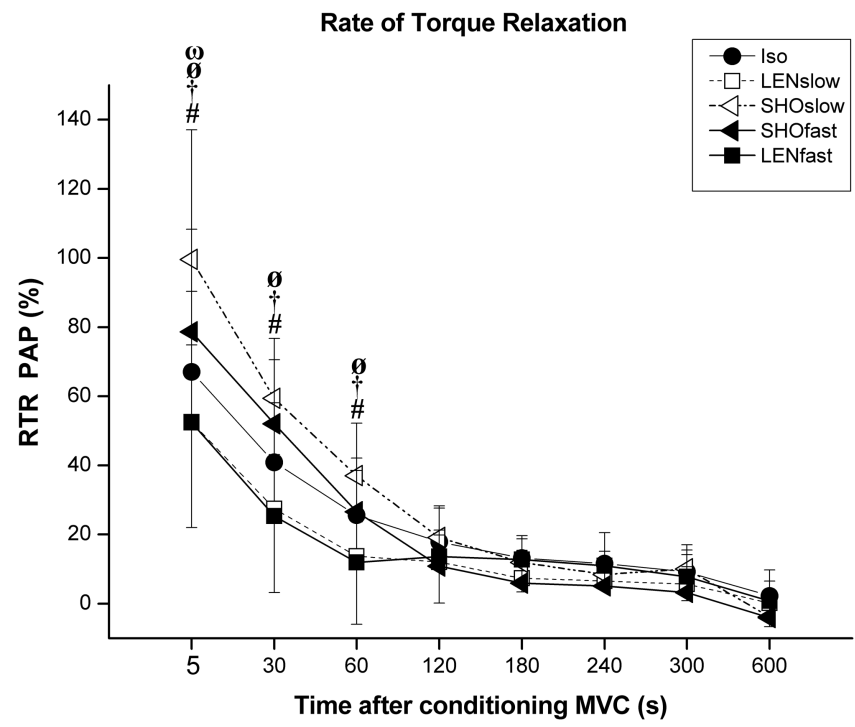

FIGURE 4-Rate of torque relaxation (RTR) PAP (\% increase from control twitch values) at different delays after the conditioning MVC for the five different modes. Significant $(P<0.05)$ differences in degree of RTR PAP are indicated by the following: \#differences between fast lengthening $\left(\mathbf{L E N}_{\text {fast }}\right)$ and fast shortening $\left(\mathbf{S H O}_{\text {fast }}\right)$, $\dagger$ differences between slow lengthening $\left(\mathrm{LEN}_{\text {slow }}\right)$ and slow shortening $\left(\mathrm{SHO}_{\text {slow }}\right)$, Ødifferences between isometric and any other mode, and wdifferences between velocities within the shortening modes.

tests showed that compared with the control twitches, the rising time ${ }_{10-90}$ was significantly decreased from $5 \mathrm{~s}$ to 1 min after the MVC for ISO, $\mathrm{LEN}_{\text {fast }}$, and $\mathrm{LEN}_{\text {slow }}$ only.

Change in rising time $10-90$ (expressed as increase in percent of control twitch values) at different delays after the conditioning MVC are displayed in Figure 5. Change in rising time $_{10-90}$ differed between modes $\left(F_{4,40}=9.1\right)$, and an

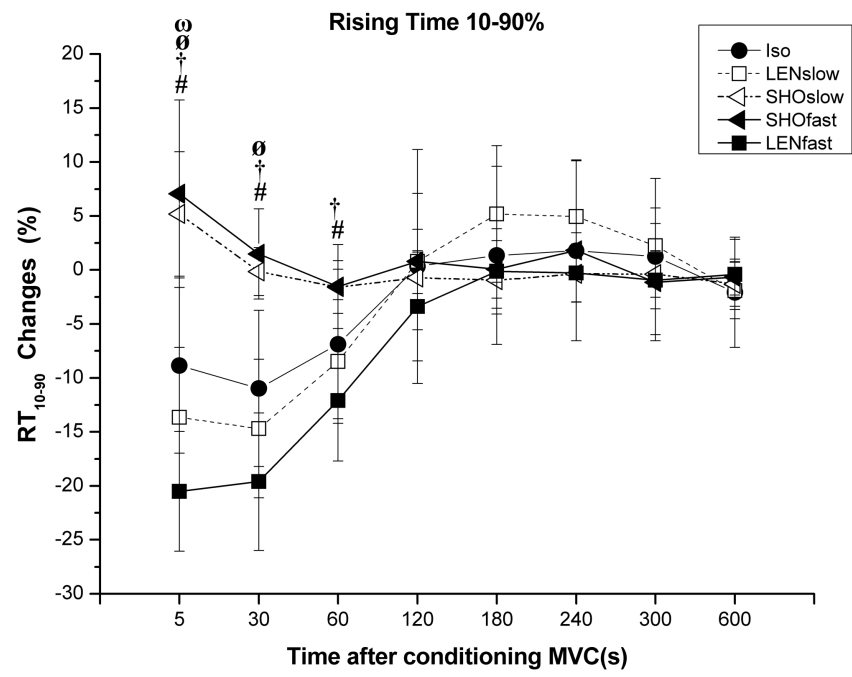

FIGURE 5-Change in rising time $\left(\mathrm{RT}_{10-90}\right)$ (expressed as increase in $\%$ of control twitch values) at different delays after the conditioning MVC for the five different modes. Significant $(P<0.05)$ differences in degree of degree of change in $\mathrm{RT}_{10-90}$ are indicated by the following: \#differences between fast lengthening $\left(\mathbf{L E N}_{\text {fast }}\right)$ and fast shortening $\left(\mathrm{SHO}_{\text {fast }}\right)$, †differences between slow lengthening $\left(\mathbf{L E N}_{\text {slow }}\right)$ and slow shortening $\left(\mathrm{SHO}_{\text {slow }}\right)$, Ødifferences between isometric and any other mode, and wdifferences between velocities within the lengthening modes. interaction was found between mode and time $\left(F_{28,280}=22.9\right)$. The mean change in rising time ${ }_{10-90}$ was rather small, ranging from $-7.1 \% \pm 8.8 \%$ in the $\mathrm{LEN}_{\text {fast }}$ to $1.0 \% \pm 2.7 \%$ in the $\mathrm{SHO}_{\text {fast }}$ mode. Post hoc tests showed significant differences between $\mathrm{LEN}_{\text {slow }}$ and $\mathrm{LEN}_{\text {fast }}$ at $5 \mathrm{~s}$ after MVC (Fig. 5) only, while for the same angular velocity, SHO and LEN values differed significantly from $5 \mathrm{~s}$ to $1 \mathrm{~min}$ after the conditioning MVC (Fig. 5). Finally, in the ISO mode, the change in rising time $_{10-90}$ was $-8.8 \% \pm 8.1 \%$, at $5 \mathrm{~s}$ and $-6.9 \% \pm 6.9 \%$ at $1 \mathrm{~min}$ after MVC. The change in rising time ${ }_{10-90}$ was lower in the ISO than the $\mathrm{SHO}_{\text {fast }}$ and $\mathrm{SHO}_{\text {slow }}$ modes for up to $30 \mathrm{~s}$ after the MVC and higher in the $\mathrm{LEN}_{\text {fast }}$ mode for up to $30 \mathrm{~s}$ after the MVC. No significant differences were identified between the ISO mode and the $\mathrm{LEN}_{\text {slow }}$ mode (Fig. 5).

HRT. ICC $_{\mathrm{c}}$ for the control twitch HRT ranged between 0.918 and 0.964 in the different modes. The absolute value of the HRT was significantly different between modes $\left(F_{4,40}=133.4\right)$, with shorter HRT for LEN than SHO. Furthermore, an interaction between time and mode $\left(F_{32,320}=22.6\right)$ was identified. Post hoc tests showed that compared with the control twitches, HRT was significantly reduced for $5 \mathrm{~min}$ in $\mathrm{LEN}_{\text {slow }}, 30 \mathrm{~s}$ in ISO and $\mathrm{SHO}_{\text {fast }}$, and for $1 \mathrm{~min}$ in $\mathrm{SHO}_{\text {slow. }}$.

The change in HRT (expressed as increase in percent of control twitch values) at different delays after the conditioning MVC are displayed in Figure 6. For change in HTR, a main effect of mode $\left(F_{4,40}=3.5\right)$ and interaction between mode and time $\left(F_{28,280}=14.1\right)$ were found. Average change in HRT was the lowest in $\operatorname{LEN}_{\text {slow }}(-9.2 \% \pm 3.8 \%)$ and the highest in $\mathrm{SHO}_{\text {fast }}(-0.1 \% \pm 7.3 \%)$. Post hoc tests showed significant differences between $\mathrm{SHO}_{\text {slow }}$ and $\mathrm{SHO}_{\text {fast }}$ from 5 to $30 \mathrm{~s}$ after the MVC (Fig. 6). HRT values during $\mathrm{SHO}_{\text {slow }}$ and LEN $_{\text {slow }}$ differed significantly from $5 \mathrm{~s}$ to $5 \mathrm{~min}$, and the same

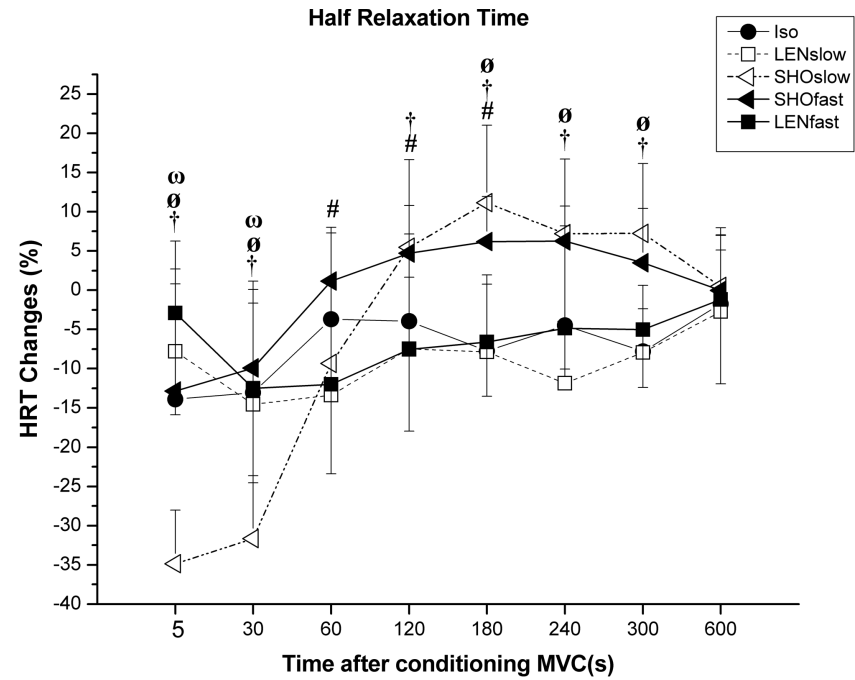

FIGURE 6-Change in half relaxation time (HRT) (expressed as increase in $\%$ of control twitch values) at different delays after the conditioning MVC. Significant $(P<0.05)$ differences in degree of change in HRT are indicated by the following: \#differences between fast lengthening $\left(\mathbf{L E N}_{\text {fast }}\right)$ and fast shortening $\left(\mathrm{SHO}_{\text {fast }}\right), \dagger$ differences between slow lengthening $\left(\mathrm{LEN}_{\text {slow }}\right)$ and slow shortening $\left(\mathrm{SHO}_{\text {slow }}\right)$, Ødifferences between isometric and any other mode, and $\omega$ differences between velocities within the shortening modes. 
parameter during $\mathrm{SHO}_{\text {fast }}$ and $\mathrm{LEN}_{\text {fast }}$ differed from 1 to $3 \mathrm{~min}$ after the conditioning MVC (Fig. 6). Finally, in the ISO mode, the change in HRT was $-13.9 \% \pm 14.6 \%$ at $5 \mathrm{~s}$ and $-13.0 \% \pm$ $13.1 \%$ at $30 \mathrm{~s}$ after the MVC, occupying a middle position in relation to $\mathrm{SHO}_{\text {slow, }}$ presenting higher values up to $30 \mathrm{~s}$ and inverting to lower values from 3 to 5 min after MVC (Fig. 6). Change in HRT in the ISO mode was not as pronounced as it was in $\mathrm{SHO}_{\text {fast }}$ mode, being significant only at 3 and 5 min after the MVC. No significant differences were found between the ISO mode and the $\mathrm{LEN}_{\text {slow }}$ and $\mathrm{LEN}_{\text {Fast }}$ modes (Fig. 6).

M-wave. $\mathrm{ICC}_{\mathrm{c}}$ of the M-waves associated with the control twitches ranged between 0.995 and 0.999 in the different modes. The absolute value of the M-wave was significantly different between modes $\left(F_{4,40}=15.6\right)$, and time $\left(F_{8,80}=2.8\right)$. However, there was no significant interaction between mode and time $\left(F_{32,320}=0.41, P=0.99\right)$. Post hoc tests showed that compared with control values the M-wave was significantly increased by $5.9 \% \pm 1.2 \%$ at $5 \mathrm{~s}$ after MVC only.

Associations between twitch properties. For all modes, the peak twitch was strongly positively correlated to the RTD and the RTR. Mode-specific correlations between peak twitch, RTD, and RTR ranged from $r=0.96$ to $r=0.99$ $(P<0.001)$. Negative correlations were identified between RTD and rising time ${ }_{10-90}$, for the ISO $(r=-0.87, P=0.04)$, $\operatorname{LEN}_{\text {slow }}(r=-0.88, P=0.04)$, and $\operatorname{LEN}_{\text {fast }}(r=-0.94$, $P<0.001)$ modes, whereas positive correlations were found for the $\mathrm{SHO}_{\text {slow }}(r=0.79, P=0.019)$ and $\mathrm{SHO}_{\text {fast }}$ $(r=0.74, P=0.032)$ modes. Between RTR and HRT, an allmode negative correlation was found $(r=-0.66, P<0.001)$, whereas for the $\mathrm{LEN}_{\text {fast }}$ mode, no relationship was present $(r=-0.005, P=0.99)$.

\section{DISCUSSION}

The main findings of the present study were that both the extent and the duration of PAP were dependent on direction and velocity of ongoing muscle length changes. Such modespecific potentiation was not found for the supramaximal M-wave. An increase in peak twitch, RTD, and RTR after a 6-s MVC was present in all modes, but the largest increase relative to the control twitch values was seen in the SHO modes. There was, however, still substantial PAP during plantarflexor muscle lengthening.

Degree and duration of isometric potentiation. Human studies on muscle contractile properties during ongoing muscle length changes are rare. Therefore, only results from the isometric mode could be fully compared between studies. Both the degree and the duration of the isometric potentiation seen in the current study were within the range of what has been demonstrated previously. In the isometric mode, the potentiation of peak twitch, RTD, and RTR lasted for 5, 2, and $3 \mathrm{~min}$, respectively. In the same muscle group, the duration of PAP has been shown to range between 5 and $10 \mathrm{~min}$ after a conditioning contraction $(27,38)$. Furthermore, the duration of potentiation does not seem to be affected by muscle group because a similar duration of potentiation was observed in both the knee extensors (31) and the tibialis anterior (3). In the isometric mode of the power athletes tested in the current study, the peak twitch increased by $67.0 \% \pm$ $21.6 \%$, RTD by $102.2 \% \pm 33.3 \%$, and RTR by of $66.9 \% \pm$ $23.5 \% 5 \mathrm{~s}$ after MVC, which seems to be greater than previously reported in most other studies $(6,13,20,36,38)$. Ereline et al. (13) and Hamada et al. (20) compared potentiation in the plantarflexors of different groups of subjects and found increases in peak twitch for sedentary, active, and athletic subjects of $20 \%-28 \% ; 25 \%-38 \%$, and $50 \%$, respectively. Other studies with a less homogenous sample of subjects have however reported rather high levels of peak twitch increases-a $68 \%$ increase in a mixed population (active and athletes) (35) and a 78\% increase in a population of active males (27). Therefore, it cannot be stated that the athletes participating in the current study have higher degrees of peak twitch potentiation than the general population. Information on the effects of PAP on RTD and RTR in the plantarflexors is limited. Of the above studies, only Shima et al. (36) reported RTD values. They found an enhancement of $86 \%$ in peak RTD, that is, less than what is presented in our study. For the quadriceps muscles of active subjects, peak twitch has been shown to increase by $48 \%$, RTD by $87 \%$, and RTR by $102 \%$ (33), that is, lesser increases of peak twitch and RTD but larger increases of RTR compared with our study. The quadriceps muscles are known to have a higher percentage of type 2 fibers compared with the triceps surae (14). A higher percentage of type 2 fibers has been associated with more phosphorylation of the regulatory light chains and larger amounts of PAP $(19,37)$. The differences seen between our data on the plantarflexors and the above-mentioned study on the quadriceps is therefore more likely related to differences in study population rather than differences in muscle group. Our isometric data further showed a significant decrease in rising time ${ }_{10-90}(-8.8 \% \pm 8.1 \%)$ and HRT $(-13.9 \% \pm 146 \%)$, which is consistent with the studies of Belanger et al. (6), Vandervoort et al. (38), and Hamada et al. (20) for contraction time and TTP twitch. Although not significant in any of the studies investigating HRT, all of them showed a visible trend toward a shorter HRT after conditioning $(20,35,38)$.

Mode dependent potentiation. In the present study, the peak twitch, RTD, and RTR increased in all modes, although to different extents between modes, with potentiation lasting up to $5 \mathrm{~min}$ (Figs. 2-4). For rising time ${ }_{10-90}$ and HRT on the other hand, only some of the modes displayed similar behavior (Figs. 5-6). Strong positive correlations were found between peak twitch, RTD, and RTR, suggesting that the mechanisms underlying these parameters changed in parallel in the investigated modes. Correlations between RTD and rising time $e_{10-90}$ were positive for shortning modes and negative for lengthening modes. This is likely due to the rise in peak twitch being greater than the rise in RTD in the shortening modes, whereas for the lengthening modes, the opposite 
was true. Most previous studies have suggested that an increase in peak twitch is associated with an increase in RTD since increased phosphorylation of the myosin regulatory light chains enhances $\mathrm{Ca}^{2+}$ sensitivity and rate of cross-bridge attachment causing an enhanced number of attached cross bridges $(7,8,15,39)$. The RTR on the other hand has often, but not always, been shown to be enhanced following a conditioning MVC (24). Macintosh (24) suggested that an increase in phosphorylation of the myosin regulatory light chains does not cause a substantial increase in the rate of cross-bridge detachment, and when a conditioning contraction enhances RTR, this could instead be linked to an increase in $\mathrm{Ca}^{2+}$ uptake. The PAP of peak twitch, RTD, and RTR were all more pronounced during muscle shortening compared with lengthening. The cross-bridge cycling mechanism has been suggested to differ between muscle lengthening and shortening (8). For example, lengthening increases the proportion of force-generating cross bridges, possibly due to a higher resistance resulting from the binding of both myosin heads to actin (9), thus causing the twitches induced during muscle lengthening to be larger. The possible causes of differences in PAP between modes include differences in the attached number of cross bridges in the unpotentiated state, structural differences in the series of elastic components $(8,18,29)$, and/or a ceiling effect of contractile properties (29), reducing the potential for PAP during ongoing muscle lengthening. As expected, RTR was negatively correlated with HRT in all modes exept for $\mathrm{LEN}_{\text {fast }}$. The reason for the lack of relationship in this mode is unclear. RTR and HRT have been suggested to be associated with the $\mathrm{Ca}^{2+}$ reuptake capacity of the sarcoplasmic reticulum, $\left[\mathrm{Ca}^{2+}\right]$ in the interfibrillar area, the capacity of troponin C to bind and release calcium $(23,24,39)$, and the modulation of cross-bridge kinetics (39). The differences between modes for the HRT and the RTR parameters were not expected and rather modest.

To the best of our knowledge, only one previous study (2) tested and compared PAP of twitches during ongoing muscle length changes in humans. Baubault et al. (2) investigated PAP during passive lengthening and shortening of the quadriceps muscle group. Our findings are not expected to be identical with those of Baubault et al. (2) because, for example, (a) different muscle groups and angular velocities were tested, (b) different subjects were used (our highly trained power athletes vs their nonathletes), and (c) different conditioning contraction protocols were implemented (our 6-s MVC at $90^{\circ}$ ankle angle vs their 3-s MVC in knee flexion or extension). Despite these differences, findings were surprisingly similar with both studies showing that potentiation was indeed present also during ongoing muscle lengthening. Baubalt et al. (2) reported $15 \%$ potentiation of peak twitch in slow $\left(30^{\circ} \cdot \mathrm{s}^{-1}\right)$ lengthening and $10 \%$ potentiation in fast $\left(150^{\circ} \cdot \mathrm{s}^{-1}\right)$ lengthening, whereas the current study reported $43 \%$ and $30 \%$ for $\operatorname{LEN}_{\text {slow }}\left(30^{\circ} \cdot \mathrm{s}^{-1}\right)$ and $\operatorname{LEN}_{\text {fast }}\left(60^{\circ} \cdot \mathrm{s}^{-1}\right)$, respectively. One possible explanation for such dissimilarities in potentiation levels may be differences in the muscle length at which the maximal voluntary conditioning contraction was performed. An MVC performed at shorter than optimal muscle length would result in less muscle activation and therefore torque (25), thereby reducing the degree of potentiation (38). The study by Babault et al. (2) may therefore have underestimated the degree of potentiation during lengthening and overestimated the degree of potentiation during shortening. Our data further showed that the decay of the induced changes behaved differently between modes for the different analyzed parameters. The difference between modes was especially evident for the rising time ${ }_{10-90}$ and HRT parameters. A few animal studies also investigated PAP under dynamic conditions $(10,16)$. Caterini et al. (10) suggested that potentiation was greater during the shortening phase than the lengthening phase in a cyclic movement and that the potentiation was greater with higher shortening velocities. Although Caterini et al. (10) reported no significant potentiation of peak twitch during ongoing muscle lengthening, we showed that in male power athletes, the potentiation affects contractile properties during ongoing lengthening, although to a lesser extent than that in ongoing muscle shortening.

Methodological considerations. To fully understand the implications of the findings presented here a few methodological difficulties need to be discussed.

In the present study, all trials were conducted for each subject in a single session at a stable room temperature. While intramuscular temperature or skin temperature were not assessed in this study, Baudry et al. (3) demonstrated that a 6-s MVC did not significantly influence skin temperature, either immediately or 20 min after a conditioning contraction (3). Furthermore, Davis et al. (12) found that muscular heating did not significantly impact the supramaximal twitch force. Therefore, it seems plausible to assume that the 6-s MVC performed by the subjects in the current study did not change their muscle temperature to a degree that might affect the data.

Another issue worth considering is that a maximal activation performed at a given muscle length could induce thixotropic and/or hysteresis-like phenomena affecting muscle twitch contractile properties if they are assessed at another muscle length. Proske and Morgan $(29,30)$ found that a slack effect was generated if a muscle contraction was induced at a higher muscle length than the one tested. However, in the present study, the conditioning MVC was performed at the same angle as where the twitches were tested. Also, in the long resting period between twitches, the foot was passively positioned into the neutral position and subsequently positioned in either the dorsi- or plantarflexion position only $30 \mathrm{~s}$ before a passive shortening or lengthening, respectively. Applying those maneuvers should have minimized any differences between modes in terms of thixotropic and/or hysteresis-like phenomena $(1,29,30)$.

Potentiation has been shown to differ between different muscle lengths $(8,24,32)$. Therefore, a pilot study was performed to gain information on how the electrical stimulation should be timed for all modes in order for the peak twitch to arrive at the neutral position. These types of adjustments, also 
performed by Gravel et al. (17), resulted in a peak twitch angle variation of $0.68^{\circ} \pm 2.24^{\circ}$ between all modes. Thus, the differences in PAP between modes in this study are unlikely to be related to variations in muscle lengths.

In the current study, the peak twitch was measured as the difference between the maximal twitch torque and the torque at the time of the proximal peak of the soleus M-wave. This could mean that the absolute values of the shortening peak twitches are slightly underestimated and that the absolute values of the lengthening peak twitches are slightly overestimated. This may in turn have induced a slight overestimation of potentiation in shortening modes and a corresponding underestimation of the potentiation in lengthening modes.

Implications. The present data, together with previous animal $(8,10,16,34)$ and controlled human studies $(2,4,5)$, suggest that PAP is action-type dependent but still present in all action types. Functional enhancements might therefore be expected to occur not only in isometric and concentric actions but also in the eccentric part of the movement. Enhanced mechanical efficiency during ongoing muscle length changes might translate into both prolonged endurance $(20,26)$ in submaximal cyclic muscle actions and into enhanced RTD and power production in explosive tasks, such as a sprint start. Further studies are required to assess if and how enhanced contractile properties seen in muscles during static and dynamic conditions can be functionally exploited for sports performance. The findings of this study also have implications for studies using the twitch interpolation

\section{REFERENCES}

1. Axelson HW, Hagbarth K-E. Human motor control consequences of thixotropic changes in muscular short-range stiffness. J Physiol. 2001;535(1):279-88.

2. Babault N, Maffiuletti NA, Pousson M. Postactivation potentiation in human knee extensors during dynamic passive movements. Med Sci Sports Exerc. 2008;40(4):735-43.

3. Baudry S, Duchateau J. Postactivation potentiation in human muscle is not related to the type of maximal conditioning contraction. Muscle Nerve. 2004;30(3):328-36.

4. Baudry S, Duchateau J. Postactivation potentiation in a human muscle: effect on the load-velocity relation of tetanic and voluntary shortening contractions. J Appl Physiol. 2007;103(4):1318-25.

5. Baudry S, Duchateau J. Postactivation potentiation in a human muscle: effect on the rate of torque development of tetanic and voluntary isometric contractions. J Appl Physiol. 2007;102(4): 1394-401.

6. Belanger AY, McComas AJ, Elder GB. Physiological properties of two antagonist human muscle groups. Eur J Appl Physiol Occup Physiol. 1983;51(3):381-93.

7. Brito R, Alamo L, Lundberg U, et al. A molecular model of phosphorylation-based activation and potentiation of tarantula muscle thick filaments. J Mol Biol. 2011;414(1):44-61.

8. Brown IE, Loeb GE. Measured and modeled properties of mammalian skeletal muscle. I. The effects of post-activation potentiation on the time course and velocity dependencies of force production. J Muscle Res Cell Motil. 1999;20(5):443-56.

9. Brunello E, Reconditi M, Elangovan R, et al. Skeletal muscle resists stretch by rapid binding of the second motor domain of myosin to actin. Proc Natl Acad Sci U S A. 2007;104(50):20114-9. technique to compare voluntary activation between action types and velocities.

\section{CONCLUSIONS}

The power athletes included in the current study showed a degree and duration of PAP that was dependent on direction and velocity of ongoing muscle length changes. The PAP of contractile properties was lower during muscle lengthening than shortening for some, but not all, of the analyzed parameters, suggesting that a conditioning MVC may enhance RTD and peak twitch not only in isometric and concentric conditions but also in eccentric muscle actions for up to approximately $5 \mathrm{~min}$. Future studies trying to link conditioning effects on contractile properties to effects on dynamic sports performance should consider measuring muscle contractile properties during ongoing muscle length changes instead of restricting to isometric conditions only.

This project was financially supported by the Swedish National Centre for Research in Sports (CIF). Paulo Gago wishes to thank the Fundação para a Ciência e Tecnologia (FCT), Portugal, for the Ph.D. Grant (SFRH/BD/79184/2011).

The authors wish to thank Olga Tarassova for her skillful assistance in data collection for this study.

No conflict of interests, financial or otherwise, are declared by the authors.

The results of the present study do not constitute endorsement by the American College of Sports Medicine.

10. Caterini D, Gittings W, Huang J, Vandenboom R. The effect of work cycle frequency on the potentiation of dynamic force in mouse fast twitch skeletal muscle. J Exp Biol. 2011;214(23):3915-23.

11. Chiu LZF, FRY AC, Weiss LW, Schilling BK, Brown LE, Smith SL. Postactivation potentiation response in athletic and recreationally trained individuals. J Strength Cond Res. 2003;17(4):671-7.

12. Davies CT, Mecrow IK, White MJ. Contractile properties of the human triceps surae with some observations on the effects of temperature and exercise. Eur J Appl Physiol Occup Physiol. 1982;49(2):255-69.

13. Ereline J, Gapeyeva H, Pääsuke M. Comparison of twitch contractile properties of plantarflexor muscles in Nordic combined athletes, cross-country skiers, and sedentary men. Eur J Sport Sci. 2011;11(1):61-7.

14. Gollnick P, Sjödin B, Karlsson J, Jansson E, Saltin B. Human soleus muscle: a comparison of fiber composition and enzyme activities with other leg muscles. Pflugers Arch. 1974;348(3):247-55.

15. Gordon AM, Regnier M, Homsher E. Skeletal and cardiac muscle contractile activation: tropomyosin "rocks and rolls." Physiology. 2001;16(2):49-55

16. Grange RW, Vandenboom R, Xeni J, Houston ME. Potentiation of in vitro concentric work in mouse fast muscle. $J$ Appl Physiol. 1998;84(1):236-43

17. Gravel D, Bélanger A, Richards C. Study of human muscle contraction using electrically evoked twitch responses during passive shortening and lengthening movements. Eur J Appl Physiol Occup Physiol. 1987;56(6):623-7.

18. Hagbarth KE, Hägglund JV, Nordin M, Wallin EU. Thixotropic behaviour of human finger flexor muscles with accompanying 
changes in spindle and reflex responses to stretch. $J$ Physiol. 1985;368(1):323-42.

19. Hamada T, Sale DG, MacDougall JD, Tarnopolsky MA. Postactivation potentiation, fiber type, and twitch contraction time in human knee extensor muscles. $J$ Appl Physiol. 2000;88(6):2131-7.

20. Hamada TS, Digby G, Duncan MJ. Postactivation potentiation in endurance-trained male athletes. Med Sci Sports Exerc. 2000;32(3): 403-11.

21. Jubeau M, Gondin J, Martin A, Van Hoecke J, Maffiuletti NA. Differences in twitch potentiation between voluntary and stimulated quadriceps contractions of equal intensity. Scand $J$ Med Sci Sports. 2010;20(1):e56-62.

22. Kilduff LP, Bevan HR, Kingsley MIC, et al. Postactivation potentiation in professional rugby players: optimal recovery. $J$ Strength Cond Res. 2007;21(4):1134-8.

23. Kugelberg E, Thornell L-E. Contraction time, histochemical type, and terminal cisternae volume of rat motor units. Muscle Nerve. 1983;6(2):149-53.

24. MacIntosh BR. Cellular and Whole Muscle Studies of Activity Dependent Potentiation Muscle Biophysics. In: Rassier DE, editor. New York: Springer; 2010. pp. 315-42.

25. Maffiuletti NA, Lepers R. Quadriceps femoris torque and EMG activity in seated versus supine position. Med Sci Sports Exerc. 2003;35(9):1511-6.

26. Mettler JA, Griffin L. Postactivation potentiation and muscular endurance training. Muscle Nerve. 2012;45(3):416-25.

27. Miyamoto N, Kanehisa H, Fukunaga T, Kawakami Y. Effect of postactivation potentiation on the maximal voluntary isokinetic concentric torque in humans. $J$ Strength Cond Res. 2011;25(1):186-92 10.1519/JSC.0b013e3181b62c1d.

28. O'Leary DD, Hope K, Sale DG. Posttetanic potentiation of human dorsiflexors. J Appl Physiol. 1997;83(6):2131-8.

29. Proske U, Morgan DL. Do cross-bridges contribute to the tension during stretch of passive muscle? J Muscle Res Cell Motil. 1999;20(5-6):433-42.
30. Proske U, Morgan DL, Gregory JE. Thixotropy in skeletal muscle and in muscle spindles: a review. Progress Neurobiol. 1993;41(6): 705-21.

31. Pääsuke M, Saapar L, Ereline J, Gapeyeva H, Requena B, Ööpik V. Postactivation potentiation of knee extensor muscles in power- and endurance-trained, and untrained women. Eur Journal Appl Physiol. 2007;101(5):577-85.

32. Rassier DE. The effects of length on fatigue and twitch potentiation in human skeletal muscle. Clin Physiol. 2000;20(6):474-82.

33. Requena B, Gapeyeva H, García I, Ereline J, Pääsuke M. Twitch potentiation after voluntary versus electrically induced isometric contractions in human knee extensor muscles. Eur Journal Appl Physiol. 2008;104(3):463-72.

34. Rijkelijkhuizen JM, de Ruiter CJ, Huijing PA, de Haan A. Lowfrequency fatigue, post-tetanic potentiation and their interaction at different muscle lengths following eccentric exercise. J Exp Biol. 2005;208(1):55-63.

35. Sasaki K, Tomioka Y, Ishii N. Activation of fast-twitch fibers assessed with twitch potentiation. Muscle Nerve. 2012;46(2):218-27.

36. Shima N, Rice CL, Ota Y, Yabe K. The effect of postactivation potentiation on the mechanomyogram. Eur Journal Appl Physiol. 2006;96(1):17-23.

37. Smith JC, FRY AC. Effects of a ten-second maximum voluntary contraction on regulatory myosin light-chain phosphorylation and dynamic performance measures. J Strength Cond Res. 2007;21(1):73-6.

38. Vandervoort AA, Quinlan J, McComas AJ. Twitch potentiation after voluntary contraction. Exp Neurol. 1983;81(1):141-52.

39. Westerblad H, Lännergren J, Allen DG. Slowed relaxation in fatigued skeletal muscle fibers of Xenopus and mouse: contribution of $\left[\mathrm{Ca}^{2+}\right] \mathrm{i}$ and cross-bridges. J Gen Physiol. 1997;109(3):385-99.

40. Zhi G, Ryder JW, Huang J, et al. Myosin light chain kinase and myosin phosphorylation effect frequency-dependent potentiation of skeletal muscle contraction. Proc Natl Acad Sci U S A. 2005;102(48): 17519-24. 